\title{
Single Stage Resection of Renal Cell Carcinoma with Right Atrial Extension-A Case Report
}

\author{
SA NAHIAN $^{\mathrm{a}}, \mathrm{S}_{\text {BISWAS }}^{\mathrm{b}}, \mathrm{R}^{\mathrm{HASSAN}}{ }^{\mathrm{c}}$, MZ HASAN $^{\mathrm{d}}$
}

\begin{abstract}
Summary:
Renal cell carcinoma (RCC) is the commonest primary tumor of the kidney which may invade through the renal vein into the inferior vena cava (IVC), and then it can extend intraluminally with subsequent tumor-thrombus formation. Here we report a case involving excision of a primary $R C C$ with tumor-thrombus involving IVC up to right atrium with the use of extracorporeal circulation.
\end{abstract}

Single stage surgical procedure was performed in collaboration with a urological team aiming complete resection of primary tumor, para-aortic lymphadenectomy and removal of IVC thrombus extending to right atrium with the help of cardiopulmonary bypass. After arresting

\section{Introduction:}

Renal cell carcinoma ( $\mathrm{RCC}$ ) is the commonest primary tumor of the kidney. RCC may invade through the renal vein into the inferior vena cava (IVC), and then it can extend intraluminally with subsequent tumor-thrombus formation, which occurring in $5 \%-15 \%$ of all cases while the tumor may extend up to the right cardiac chambers in $1 \%$ of cases ${ }^{1}$.

According to Sweeney et $\mathrm{al}^{2}$ there are 4 stages of cavoatrial tumor-thrombus extension: in type I the intravascular tumor has occupied the renal vein but not reached the IVC, in type II the IVC is involved up to the level of the hepatic veins, while in types III and IV

1. Dr. Syed Al-Nahian, Specialist Cardiac Surgeon, Department of Cardiac Surgery, United Hospital Limited, Gulshan, Dhaka.

2. Dr. Sonjoy Biswas, Specialist Cardiac Surgeon, Department of Cardiac Surgery, United Hospital Limited, Gulshan, Dhaka.

3. Dr. Rezaul Hassan, Consultant Cardiac Surgeon, Department of Cardiac Surgery, United Hospital Limited, Gulshan, Dhaka.

4. Dr. M Zahid Hasan, Consultant, Department of Urology, United Hospital Limited, Gulshan, Dhaka.

Address of Correspondence: Dr.Sonjoy Biswas, Specialist Cardiac Surgeon, Department of Cardiac Surgery, United Hospital Limited, Dhaka, Mobile: 01713062204

e-mail-sanjoy.tapoo@gmail.com

Received: 11 Oct. 2017 heart, $R A$ was opened and the mass was removed through RA from IVC and hepatic vein level. Abdominal IVC was opened and the entire residual mass was removed from below also small amount of thrombus removed from left renal vein.

Postoperative venous doppler showed no residual thrombus in venous system. Histopathology report confirmed papillary renal cell carcinoma. The patient was discharged from hospital in the $12^{\text {th }}$ post-operative day without any complication.

(J Bangladesh Coll Phys Surg 2018; 36: 77-79) DOI: http://dx.doi.org/10.3329/jbcps.v36i2.36070

the supradiafragmatic IVC and the right cardiac chamber are involved. In type I and type II radical nephrectomy with excision of the tumor may be done without extracorporeal circulation but cases of type III and IV requires the use of extracorporeal circulation.

In the absence of local infiltration or metastatic disease intra vascular extension does not affect the prognosis adversely, provided complete resection is achieved ${ }^{1}$. Here we report a case involving excision of a primary RCC with tumor-thrombus involving IVC up to right atrium (type IV) with the use of extracorporeal circulation.

\section{Case report:}

A 48 years old hypertensive female presented with 2 months old history of generalized weakness, loss of appetite, weight loss and abdominal discomfort. On physical examination, patient was anemic, icteric with mild tachycardia. In her laboratory examination, anemia, elevated level of serum bilirubin and liver enzymes (ALT, AST, Alk Phos), raised serum LDH, features of coagulopathy (raised APTT, INR, FDP, D-dimer) were found. Abdominal ultrasonography (USG) showed right renal mass, dilated IVC with echogenic thrombus in right renal vein and IVC extending up to the heart. Contrast CT scan of the whole abdomen and chest showed right 
kidney is replaced by a heterogenous density soft tissue mass with extension in right renal vein, IVC, proximal part of left renal vein, confluence of hepatic vein and up to the right atrium (RA) (Fig-1).

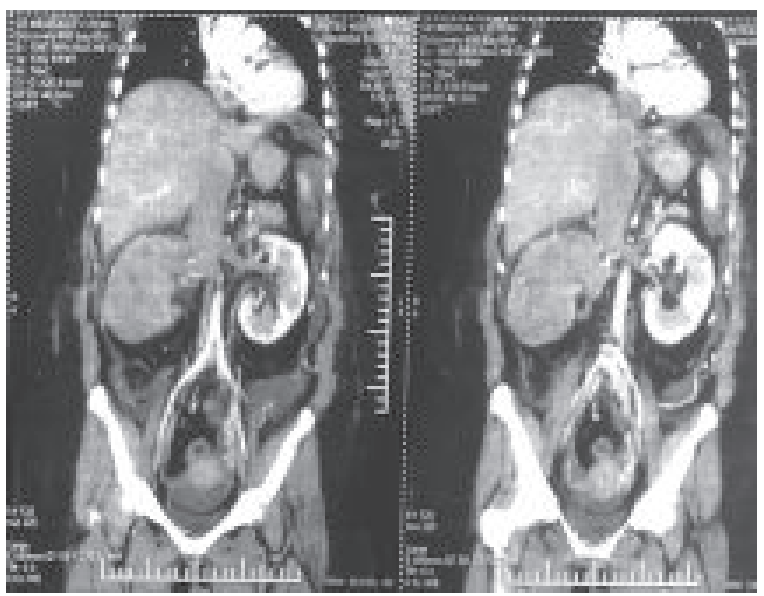

Fig.-1: $C T$ scan showing tumor thrombus (arrow) extending to IVC up to $R A$

There were no hepatic lesion and no para aortic lymphadenopathy. There were no lesion found in intrathoracic great vessels and lungs.

One stage surgical procedure was planned in collaboration with a urological team aiming complete resection of primary tumor, para-aortic lymphadenectomy (if present) and removal of IVC thrombus extending to RA with the help of cardiopulmonary bypass. The right kidney was mobilized by sharp and blunt dissection through a rooftop incision. IVC felt firm with tumor thrombus within. Right nephrectomy was performed. IVC was mobilized gently and taped. Cardiopulmonary bypass (CPB) was established by aortic cannula (24 Fr), venous cannulas were placed in the superior vena cava directly (24 Fr) and right femoral vein (22 Fr). Under moderate hypothermia and low flow, the ascending aorta was cross-clamped and cold blood cardioplegic solution was administered for myocardial protection antegradely. After arresting heart RA was opened and the mass was seen just reaching the IVC-RA junction (Fig-2).

The tumor thrombus was removed through RA from IVC and hepatic vein level. Abdominal IVC was opened and the entire residual mass was removed from below also small amount of thrombus removed from left renal vein. After IVC and RA were closed, the patient was gradually weaned from bypass and cannulas were removed. Total bypass time was 155 minutes and total cross clamp time was 85 minutes.

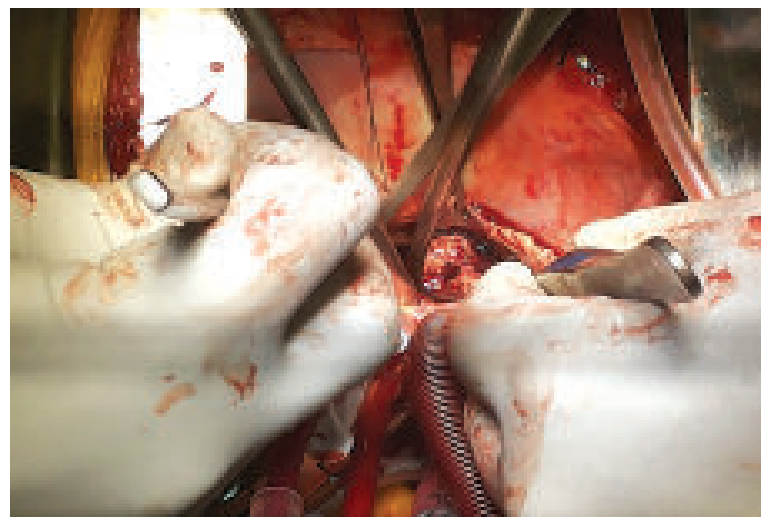

Fig.-2: Showing tumor mass (arrow) in the IVC-RA junction

Postoperative venous Doppler showed no residual thrombus in venous system. The specimen (Fig- 3) showed involvement of the perinephric fat as well as ureteric lumen. Histopathology report confirmed papillary renal cell carcinoma of the right kidney with renal capsule infiltration having TNM stage-T3b Nx, Mx. Patient did not have any complications such as pneumonia, wound infection, deep venous thrombosis rather improvement of hepatic dysfunction in the immediate post-operative period. The patient was discharged from hospital in the $12^{\text {th }}$ post-operative day. FDG based PET scan done 9 weeks after surgery which showed hyper metabolic spot only in $6^{\text {th }}$ segment of liver, IVC and left renal vein. She was further planned for targeted therapy with Pazopanib as her follow up treatment.

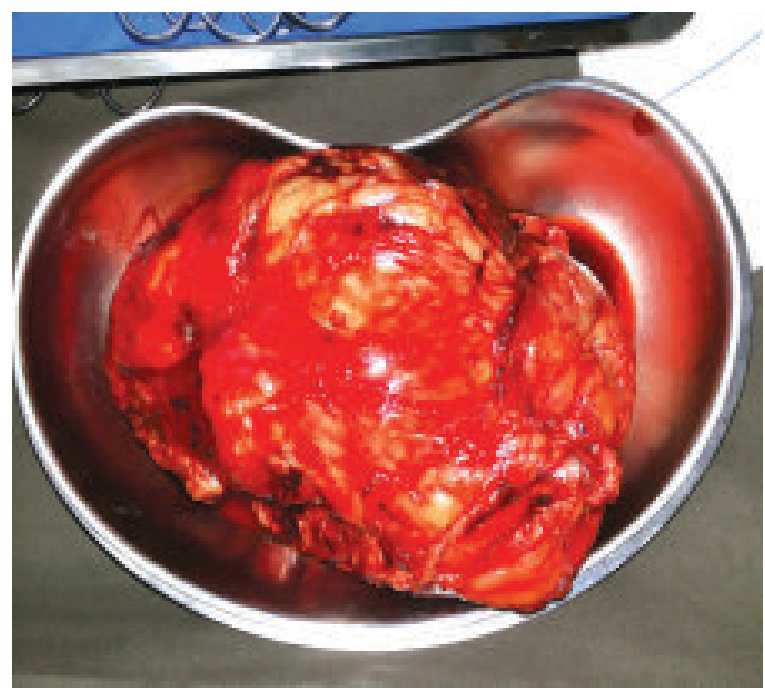

Fig.-3: Showing resected specimen of right kidney 


\section{Discussion:}

In RCC, nephrectomy and vena caval thrombectomy that extended into the IVC was first described by, Berg et al in 1913. Presence of intra vascular extension of RCC up to IVC and RA sounds a relative hopeless situation but the current available data indicates a different scenario. Presence of perinephric infiltration of the tumor with disruption of renal capsule, local lymph node involvement and distant metastasis all have a profound influence on disease-free and overall survival. However, the intravascular tumor invasion to whatever degree is not associated with an adverse prognosis, provided complete resection (R0) is achieved ${ }^{1}$. Radical nephrectomy with venacaval thrombectomy has operative mortality rates ranging from $2.7 \%$ to $13 \%$ and an expected 5 year survival ranging from $30 \%$ to $72 \%{ }^{3}$. Ioannis and coworkers ${ }^{4}$ reported that prognosis ( 5 year survival is $50 \%-68 \%$ ) is very good for a stage IIIa tumor (with no lymph or distal metastases). However, incomplete tumor resection is associated with poor prognosis ( 5 year survival: 10\%-17\%). In 1989, Skinner and coworkers et al also reported that tumor-thrombus, regardless of the degree of extension, if without metastasized local nodes or perinephric fat involvement, has a 5 year survival rate similar to that for a tumor that remains inside the renal capsule. In our patient, complete removal of the tumor was done successfully, though there was tumor cell involvement of the perinephric fat, there was no involvement of local lymph nodes and no gross evidence of distant metastasis found. Moreover, RCC is usually not responsive to conventional chemotherapy or radiotherapy, as a result surgery is the most effective method to eradicate this tumor as early as possible.

The application of CPB here aids in control of blood loss, decreases chances of distal embolism, provides adequate clean exposure to achieve total retrieval tumor thrombus from- IVC, part of hepatic vein and right atrium. Noguchi K et al ${ }^{5}$ reported the use of CPB in combination with hypothermic circulatory arrest (HCA) for complete tumor- thrombus excision. In another report Raghuram et $\mathrm{al}^{6}$ used CPB with moderate hypothermia and low flow for removal of tumor thrombus. In our case we also used CPB with low flow and could achieve adequate clearance of the tumor thrombus without any complication. Commonly the tumor thrombus of RCC does not invade the IVC as a result it can be easily peeled off the IVC wall ${ }^{7}$. Nevertheless preoperative or intraoperative transesophageal echocardiography (TEE) can be helpful to provide accurate information regarding adherence of the thrombus with the IVC or hepatic vein.

\section{Conclusion:}

The aggressive one stage surgery should be the choice of treatment for RCC with intravascular extension and can be carried out safely with the help of cardiopulmonary bypass and a combined team effort.

\section{References:}

1. Chiappini B, Savini C, Marinelli G, Suarez SM, Di Eusanio M, Fiorani V, et al. Cavoatrial tumor thrombus: single-stage surgical approach with profound hypothermia and circulatory arrest, including a review of the literature. J Thorac Cardiovasc Surg 2002; 124: 684-688.

2. Sweeney P, Wood CG, Pisters LL, Tanaka H. Surgical management of renal cell carcinoma associated with complex inferior vena caval thrombi. Urol Oncol 2003; 21: 327-333.

3. Dedeilias P, Koletsis E, Rousakis AG, Kouerinis I, Zaragkas S, Grigorakis A, et al. Deep hypothermia and circulatory arrest in the surgical management of renal tumors with cavoatrial extension. J Card Surg 2009; 24: 617-623.

4. Ioannis V, Panagiotis S, Anastasios A, Michael B, Anastasios S, Anastasios T, et al. Tumor extending through inferior vena cava into the right atrium. A late recurrence of renal cell carcinoma. Int J Cardiovasc Imaging 2003; 19: 179-182.

5. Noguchi K, Hori D, Nomura Y, Tanaka H. Renal cell carcinoma with tumor-thrombus extension into the right ventricle. Ann Vasc Surg 2012; 5, No.3: 376-380.

6. Raghuram RA, Kumar S, John VE, Raghunathan R, Balamurugan $\mathrm{K}$. One stage surgical treatment of renal cell carcinoma with extension upto right atrium - A case report. IJTCVS 2007; 23 : 153-155.

7. Jibiki M, Iwai T, Inoue Y, Sugano N, Kihara K, Hyochi N, et al. Surgical strategy for treating renal cell carcinoma with thrombus extending into the inferior vena cava. J Vasc Surg. 2004;39(4): 829-835. 